often constrain patient autonomy. Home hospice care is a subset of medical care that has consciously developed a philosophical and practical approach to encouraging patient and family engagement. This research examines how home hospice care provides a different strategy for improving patient engagement and shared decision-making. Methods: This ethnographic study draws on a sample of 55 home hospice participants, including patients, family members/caregivers, staff, and volunteers, and uses in-depth interview and observation of home hospice work to examine the process of providing and receiving hospice care. Results: I find that macro level hospice institutional structures and micro level daily work practices embody a holistic approach which assumes patients and family members are the critical experts in most instances of decision-making. Differences in institutional structure, such as providing care in patient homes and having an interdisciplinary team approach focused on the "whole person", empower the patient and family members. Likewise the micro level interactions between hospice workers and patients and family members narrow the field of purely "medical" decisions and broaden the field of decisions open to patients. Hospice workers accomplish this re-conceptualization by framing many medical decisions as being more about what is best for the patient and family and less about medical expertise. Conclusions: By broadening and re-conceptualizing the idea of decision-making, hospice workers enable patients and their families to have more control over their medical care and in many cases the process of dying itself. While some attributes of home hospice care are unique, many techniques could be introduced or accentuated in other models of medical care.

Keywords: Hospice; Decision-making

doi:10.3121/cmr.2014.1250.b3-2

B3-3:

\section{PCORI's National Clinical Research Network}

Kimberly Lane'; Jeffrey Brown'; Rachael Fleurence'; Matthew Gillman'; Sarah Greene ${ }^{2}$; Chelsea Jenter ${ }^{1}$; Karin Johnson ${ }^{3}$; Eric Larson ${ }^{3}$; Katherine Newton $^{3}$; Richard Platt ${ }^{1}$; Beth Syat ${ }^{1}$; Ella Thompson ${ }^{3}$

${ }^{1}$ Harvard Pilgrim Health Care; ${ }^{2}$ Patient-Centered Outcomes Research Institute; ${ }^{3}$ Group Health Research Institute

Background/Aims: In September 2013 the Patient-Centered Outcomes Research Institute (PCORI) funded the National Clinical Research Network (NCRN) Coordinating Center (CC) to manage the development of PCORI's national data infrastructure to support patient-centered comparative effectiveness research. Initial NCRN membership will include 8 Clinical Data Research Networks (CDRNs) and up to 18 Patient Powered Research Networks (PPRNs). A number of HMORN members submitted CDRN applications. The CDRN and PPRN applications are under review to be announced in December. (Note: the HMORN authors of this abstract have no knowledge of nor will they participate in the CDRN / PPRN selection process). Methods: The NCRN CC is led by the Harvard Pilgrim Health Care Institute, and co-led by Group Health Research Institute, the Duke Translational Medical Institute, AcademyHealth, the Brookings Institution, the Center for Medical Technology Policy, the Center for Democracy \& Technology, Johns Hopkins Berman Institute on Bioethics, and America's Health Insurance Plans. The RAND Corporation is the independent evaluator. The NCRN will organize its work around several taskforces, including: (a) Health systems interactions, led by Group Health Research Institute; (b) Creation of PCORInet, the networking capability to query NCRN partner data, led by Harvard Pilgrim Health Care Institute; (c) Governance, including how the NCRN will partner with external researchers to access the data infrastructure; and (d)Patient and stakeholder engagement, privacy, ethics and regulatory, rare disease and obesity cohorts, biorepositories, patient-reported outcomes and clinical trials. Results: PCORI's vision for the NCRN is to unite patients, healthcare systems and researchers to support rapid, effective observational and interventional studies of topics that are chosen by all stakeholders working in concert. We will describe the NCRN, the HMORN contribution to the Coordinating Center and, if applicable, will invite participation of HMORN members who are CDRN partners. Conclusions: The PCORI NCRN is advancing the nation's capacity to conduct comparative clinical effectiveness research by establishing an innovative and representative national research infrastructure. HMORN investigators lead several aspects of this cutting-edge work.

Keywords: Patient-centered; Comparative effectiveness research doi:10.3121/cmr.2014.1250.b3-3

B3-4:

Establishing an Aging-In-Place Model for the Lesbian, Gay, Bisexual, and Transgender (LGBT) Community: Lessons Learned from a Community-research Partnership

Leslie Wright ${ }^{1}$; Cathy Grimm ${ }^{2}$; Jessica Haxton Retrum ${ }^{3}$; Kenneth Helander ${ }^{4}$; Shari Wilkins; ${ }^{5}$ Jennifer Boggs ${ }^{1}$; Jennifer Dickman-Portz'; Wendolyn Gozansky $^{1}$; Diane King ${ }^{6}$

${ }^{1}$ Kaiser Permanente Colorado; ${ }^{2}$ Jewish Family Service of Colorado; ${ }^{3}$ Metropolitan State University of Denver; ${ }^{4}$ AARP Alaska; ${ }^{5}$ GLBT Services Center of Colorado; ${ }^{6}$ University Alaska Anchorage

Background/Aims: Lesbian, Gay, Bisexual and Transgender (LGBT) seniors face barriers to accessing social services. We describe formation of an interdisciplinary community-research partnership called SUSTAIN (Seniors Using Supports To Age In Neighborhoods), whose ultimate goal is to develop and adapt an aging-in-place model in a Denver metropolitan neighborhood that is home to a high concentration of LGBT individuals. Methods: Established in 2009, SUSTAIN partners represent a cross-segment of service deliver, community building, education, and research interests including social service agencies; local LGBT community members; the Denver Gay, Lesbian, Bisexual, \& Transgender Commission; a regional affiliate of Services and Advocacy for Gay, Lesbian, Bisexual, \& Transgender Elders (SAGE); the LGBT community center for the state of Colorado; an affirming church leader; an area agency on aging; a non-profit healthcare and research organization; and a university researcher specializing in aging. Discussions were conducted among partners and written comments were solicited to understand community experience and reflections on the partnership. Results: The success of our SUSTAIN partnership hinged on several features. First, most partners' affiliations bridged at least two of the aging, LGBT, and research communities we were uniting. Second, the partnership development mechanism required at least fifty-percent of funds be allocated to community partners, which was crucial for establishing trust. Third, SUSTAIN's core values embraced a commitment to collaborative principles and explicitly addressed power imbalances. Conclusions: Members with existing capacity to connect communities and formalized funding frameworks that emphasize equity may be key ingredients for growing a sustainable community-research partnership.

Keywords: LGBT seniors; Community based participatory research (CBPR) doi:10.3121/cmr.2014.1250.b3-4

PS1-23:

Prospective Association of Patient Activation Measure (PAM) with Medical Costs among Hypertension Population

David Mosen ${ }^{1}$; Judith Hibbard ${ }^{2}$; Kristal Rust ${ }^{1}$; Michael Leo ${ }^{1}$

${ }^{1}$ Kaiser Permanente Northwest; ${ }^{2}$ University of Oregon

Background/Aims: The patient activation measure (PAM) refers to an individual's skills, knowledge, and confidence to manage their health and their ability to engage health providers in shared decision making practices. Such skills are important for the ongoing management of chronic conditions, such as hypertension. Although research has examined the association of PAM with utilization and health outcomes; little research has examined the association of PAM with medical costs. The objective of this research was to examine the prospective association of PAM with four cost measures: 1) total costs, 2) emergency department (ED) costs, 3) inpatient costs and 4) pharmacy costs. Methods: Using an observational study design, we studied 1,812 patients with hypertension with a PAM assessment between 12/4/2007 and 2/28/201.The PAM is 13 items and categorizes PAM into four levels: PAM 1(lowest level of activation, where patients typically lack confidence and self-management skills and may not understand the need to be actively involved in managing their health) and PAM 4 (highest activation where patients typically have the necessary self-management skills and are more pro-active about their health). Patients received care in Kaiser Permanente Northwest (KPNW) and were enrolled in care management programs. Per 